\title{
Kernos
}

Revue internationale et pluridisciplinaire de religion grecque antique

22 | 2009

Varia

\section{Revues des revues}

\section{Vinciane Pirenne-Delforge}

\section{OpenEdition \\ Journals}

\section{Édition électronique}

URL : http://journals.openedition.org/kernos/1833

DOI : 10.4000/kernos. 1833

ISSN : 2034-7871

\section{Éditeur}

Centre international d'étude de la religion grecque antique

\section{Édition imprimée}

Date de publication : 1 janvier 2009

Pagination : 353-358

ISSN : 0776-3824

\section{Référence électronique}

Vinciane Pirenne-Delforge, "Revues des revues », Kernos [En ligne], 22 | 2009, mis en ligne le 09 septembre 2011, consulté le 21 septembre 2020. URL : http://journals.openedition.org/kernos/1833 ; DOI : https://doi.org/10.4000/kernos.1833

Ce document a été généré automatiquement le 21 septembre 2020.

Kernos 


\title{
Revues des revues
}

\author{
Vinciane Pirenne-Delforge
}

1 AKKAN Yllmaz, MALAY Hasan, « The Village Tar(i)gye and the Cult of Zeus Tar(i)gyenos in the Cayster Valley ", EA 40 (2007), p. 16-22.

2 AмIтAY Ory, "Why did Alexander the Great besiege Tyre?", Athenaeum 96 (2008), p. 91-102 [réflexion sur les motivations politico-religieuses du siège: Alexandre, descendant d'Héraclès, prétendait sacrifier à Héraclès-Melqart lors du rituel de la «résurrection» du dieu, ce qui avait de fortes implications religieuses, mais aussi politiques auxquelles les Tyriens ne pouvaient souscrire].

3 ANAGNostou-laoutides Eva, Konstan David, «Daphnis and Aphrodite: A Love Affair in Theocritus Idyll $1 »$, AJPh 129 (2008), p. 497-527 [l'objet de la passion de Daphnis serait Aphrodite elle-même].

4 AUPERT Pierre, "Nouveaux documents sur le culte d'Aphrodite à Amathonte. I. Aphrodite, l'empereur Titus et le hiéron dans les stèles: un nouveau sanctuaire amathousien d'Aphrodite ", BCH 130 (2006), p. 83-99.

5 AVRONIDAKI Christina, «Boeotian Red-Figure Imagery on Two New Vases by the Painter of the Dancing Pan», AK 51 (2008), p. 8-22 [l'un des vases représente le sacrifice imminent d'un chien à une divinité de l'enfantement, l'autre une scène de musique au gynécée].

6 BaChVArova Mary R., "Suppliant Danaids and Argive Nymphs in Aeschylus », CF 104 (2009), p. 289-310 [la menace de pollution en raison de leur suicide annoncé, et donc le caractère déviant de leur supplication, ferait allusion au rôle des Danaïdes comme nymphes argiennes, liées à l'humidité et à la fertilité du territoire].

7 BERNABÉ Alberto, "Some Thoughts about the 'New' Gold Tablet from Pherai », ZPE 166 (2008), p. 53-58 [plaidoyer en faveur de l'ancrage orphique de ce document qui évoque Déméter Chthonia et la Mètèr Oreia].

8 Bonazzi Mauro, «L'offerta di Plutarco. Teologia e filosofia nel De E apud Delphos (capitoli 1-2)», Philologus 152 (2008), p. 205-211 [le dialogue est moins un essai de résoudre l'énigme delphique qu'un hommage à la supériorité divine]. 
BORGERS Olaf, «Religious Citizenship in Classical Athens. Men and Women in Religious Representations on Vase-painting ", BABesch 83 (2008), p. 73-97 [la participation aux rituels est un élément constitutif de la citoyenneté et l'étude de la présence respective des hommes et des femmes dans les représentations à portée religieuse contredit certaines idées reçues].

BRUIT Louise, « Le religieux et le politique en Grèce ancienne (II). Déméter et Koré dans le panthéon athénien », LEC 75 (2007), p. 37-52.

11 BUDIN Stephanie Lynn, «Simonides' Corinthian Epigram », CPh 103 (2008), p. 335-353 [reprise du dossier de la supplication à Aphrodite par les femmes corinthiennes à la veille de la bataille de Salamine, déjà présent dans l'ouvrage de l'A. The Myth of Sacred Prostitution, Cambridge, 2008].

BURKERT Walter, «Pleading for Hell: Postulates, Fantasies, and the Senselessness of Punishment ", Numen 56 (2009), p. 141-160 [l'enquête porte sur les différentes manières dont les sociétés antiques ont développé un savoir sur l'enfer, dont la Grèce].

CASSIMATIS Hélène, «Éros en Italie méridionale. Approche iconographique à travers les représentations italiotes", Pallas 76 (2008), p. 51-65 [on y observe que le dieu se transforme au contact des exigences culturelles des acheteurs, qui sont différentes elles aussi selon les régions].

CHERUBINI Laura, «The Virgin, the Bear, the Upside-Down Strix: An Interpretation of Antoninus Liberalis 21 », Arethusa 42 (2009), p. 77-97.

CHESHIRE Keyne, « Kicking $\varphi \theta$ óvoc: Apollo and his Chorus in Callimachus' Hymn 2 », CPh 103 (2008), p. 354-373 [c'est la performance chorale des jeunes gens qui est le point central de la narration et l'ancrage rituel de la performance est essentiel pour la compréhension de l'hymne].

CHIAI Gian Franco, «Il villaggio e il suo dio: considerazioni sulla concorrenza religiosa nelle comunità rurali dell'Asia Minore in epoca romana ", Mythos 1 (2006-2007), p. 137-163 [les divinités honorées dans les sanctuaires ruraux de Lydie et de Phrygie sont représentées comme des monarques tout-puissants, dont le pouvoir est intimement lié au territoire, ce qui engendre une concurrence entre les cultes].

17 Coluins Derek, "The Magic of Homeric Verses », CPh 103 (2008), p. 211-236 [étude des principes sur lesquels se fondait l'usage des vers homériques à des fins magiques et donc de la portée de leur apparition dans d'autres contextes qui ne sont pas directement associés à la magie].

ColuINs Derek, «Mapping the Entrails: The Practice of Greek Hepatoscopy », AJPh 129 (2008), p. 319-345.

19 COLOMBert Ph., "La 'stèle de Saïs' et l'instauration du culte d'Arsinoé II dans la chôra », Ancient Society 38 (2008), p. 83-101 [le culte de la reine a d'abord été instauré à Alexandrie et puis seulement dans la chôra, ce qui conduit à relativiser la volonté de mêler population grecque et population égyptienne qui aurait animé Ptolémée Philadelphe dans l'instauration de ce culte].

DASEN Véronique, «Le secret d'Omphale", RA (2008), p. 265-281 [analyse de gemmes magiques où la figure d'Omphale, enceinte et pourvue des armes d'Héraclès, sert à protéger la sexualité féminine et la grossesse des forces mauvaises symbolisées par un âne]. 
D’ALFonso Francesca, « La Terra Desolata. Osservazioni sul destino di Bellerofonte (Il. 6, 200-202) », MH 65 (2008), p. 1-21.

DELATTRE Charles, « Entre mortalité et immortalité : l'exemple de Sarpédon dans l'Iliade », RPh 80 (2006), p. 259-271 [la mort de Sarpédon est l'occasion pour l'aède de mettre en scène tous les termes de l'alternative, sans chercher à les résoudre].

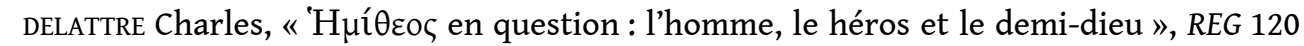
(2007), p. 481-510 [impossibilité de caractériser de façon générale et unique du demidieu dès que l'on passe d'un usage collectif, qui s'applique à un groupe d'être du temps passé, à des destinées individuelles qui font éclater l'antinomie mortalité / immortalité; annexe sur la formation du mot].

DESPINIS Giorgos I., « Neues zu der spätarchaischen Statue des Dionysos aus Ikaria », $\operatorname{MDAI}(A) 122$ (2007), 103-137 [reconstitution de la statue assise sous un baldaquin et interprétation de l'ensemble comme statue de culte].

DREYER Boris, «Le culte civique d'Arès et le panthéon de Métropolis (Ionie) », REA 110 (2008), p. 403-416 [la cité d'Ionie, pour préserver son identité spécifique, avait choisi comme dieu tutélaire Arès, d'origine indigène; de nombreuses inscriptions $d u \mathrm{I}^{\mathrm{er}} \mathrm{s}$. ap. J.-C. permettent de reconstituer l'organisation du culte et les conditions sociales et politiques de la ville].

ERICKSON Brice, «Roussa Ekklesia, Part 1: Religion and Politics in East Crete », AJA 113 (2009), p. 353-404 [analyse des ex-voto (c. 630-450) qui évoquent des rites de passage masculins liés à une déesse de source associée à la croissance des végétaux et des humains, représentée nue et portant un polos ( $\mathrm{au} \mathrm{VII}^{\mathrm{e}} \mathrm{s}$.); étude des implications politiques et sociales du culte].

FARAONE Christopher A., « Mystery Cults and Incantations. Evidence for Orphic Charms in Euripides' Cyclops 646-48? », RhM 151 (2008), p. 127-142 [si ce passage est bien fondé sur une tradition orphique, il fournit un intéressant témoignage de la forme linguistique des incantations orphiques, de même que de leur contenu narratif, à savoir le triomphe sur les forces du chaos].

8 FARAONE Christopher A., «Stopping Evil, Pain, Anger, and Blood: The Ancient Greek Tradition of Protective Iambic Incantations ", GRBS 49 (2009), p. 227-255 [d'autres mètres que l'hexamètre ont pu être utilisés à des fins de protection; étude de cette tradition et des occasions ou des lieux de son utilisation].

FEDERICO Eduardo, « Hektor sull'Isola dei Beati. Memorie e realia tebani da Licofrone a Pausania ", IncidAntico 6 (2008), p. 253-271 [histoire et évolution du culte d'Hector à Thèbes et des traditions qui s'y rapportent depuis la fin du $\mathrm{VI}^{\mathrm{e}}$ siècle av. J.-C. jusqu'à l'époque romaine].

FERRARI F., «Per leggere le lamine misteriche (I \& II), Prometheus 34 (2008), p. 1-26, 97-112.

1 FINKELBERG Margalit, "Plato, Apology 28d6-29a1 and the Ephebic Oath », SCI 37 (2008), p. 9-15 [si l'allusion au serment est bien présente dans ce passage de Platon, cela signifie qu'il remonte bien plus haut que l'année 334/3 habituellement invoquée].

FONTANNAZ Didier, «L'entre-deux-monde. Orphée et Eurydice sur une hydrie protoitaliote du sanctuaire de la source à Saturo ", AK 51 (2008), p. 41-72 [sur l'hydrie, l'espace du passage aux Enfers se confond avec celui de transition au stade de numphê et l'accent est mis sur le personnage d'Eurydice; le document fait apparaître des 
correspondance entre sphères matrimoniale et chthonienne, qui s'affirment comme des caractéristiques spécifiques de la Grande Grèce].

GAIFMAN Milette, «Visualized rituals and dedicatory inscriptions on votive offerings to the Nymphs ", Opuscula. Annual of the Swedish Institutes at Athens and Rome 1 (2008), p. 85-103 [les dédicaces accompagnées de reliefs figurant des rituels doivent être envisagées comme une expression de la piété des individus plutôt que comme le reflet de pratiques cultuelles véritables].

GARVEY Tom, "Plato's Atlantis Story: A Prose Hymn to Athena", GRBS 48 (2008), p. 381-392 [l'article met en évidence de la dimension religieuse du Timée et du Critias et considère que ces éléments créent un hymne à Athéna et contribuent à remodeler la mythologie athénienne afin de recentrer Athènes sur le mode de vie qui l'a conduite à la domination du monde]. GIANNOBILE Sergio, JORDAN D.R., " On the Text of the Hipponium Tablet », GRBS 48 (2008), p. 287-294 [reconstitution de l'archétype ionien du vers 1 de la tablette en dialecte dorien].

GIVEN John, "When Gods don't appear: divine absence and human agency in Aristophanes ", CW 102 (2009), p. 107-127.

GONZALES Matthew, "New Observations on the Lindian Cult-Tax for Enyalios ", ZPE 166 (2008), p. 121-134 [réédition de cette remarquable inscription et commentaire sur

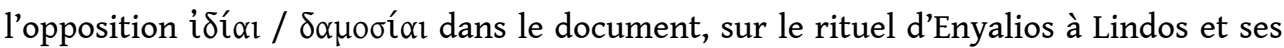
relations avec le culte du dieu ailleurs dans le monde grec]. HALUszKa Adria, « Sacred Signified: The Semiotics of Statues in the Greek Magical Papyri ", Arethusa 41 (2008), p. 479-494. HENRICHS Albert, "Reinhold Merkelbach über antike Religion, Literatur und Mysterien », ZPE 163 (2007), p. 17-24. HERMARY Antoine, « Nouveaux documents sur le culte d'Aphrodite à Amathonte. II. La tête en marbre : Aphrodite Kupria ? », BCH 130 (2006), p. 101-115.

DE LA VEGA Maria José, "Voix soumises, pratiques transgressives. Les magiciennes dans le roman gréco-romain", DHA 34 (2008), p. 27-43 [analyse de l'importance de la magie pratiquée par et pour des femmes dans les relations de genre et comme manière de produire des valeurs culturelles alternatives en marge de la culture dominante et de l'ordre social établi]. HIRSCHBERGER Martina, «Die Parteiungen der Götter in der Ilias. Antike Auslesung und Hintergründe in Kult und epischer Tradition », WS 121 (2008), p. 5-28 [c'est l'ancrage cultuel des divinités qui permettrait de comprendre pourquoi telle divinité soutiendrait tel groupe engagé dans le conflit]. HIRSCHMANN Vera, « Zwischen Menschen und Göttern. Die kleinasiatischen Engel », EA 40 (2007), p. 135-146. HÖGEMANN Peter, OETTINGER Norbert, «Die Seuche im Heerlager der Achäer vor Troia. Orakel und magische Rituale im hethiterzeitlichen Kleinasien und im archaischen Griechenland ", Klio 90 (2008), p. 7-26 [le poète de l'Iliade a vécu en Asie Mineure vers 700 et a pu faire l'expérience de pratiques remontant aux Hittites, comme la consultation oraculaire et des rituels magiques inconnus en Grèce; Crésus, redevable de la tradition anatolienne, a donc échoué à Delphes]. 

[les deux temples laissent entrevoir des traits muséaux, mais de nature différente, et sans que cela ne leur ôte leur dimension cultuelle, comme on l'a parfois prétendu pour l'Héraion].

\section{p. 275-292.} (2008), p. 22-26 [commentaire d'une nouvelle inscription mutilée mise au jour dans le sanctuaire athénien d'Aglauros et qui autorise quelques hypothèses sur les conflits rituels entre les genè en charge des cultes acropolitains, et notamment les rites vestimentaires pour Athéna, et met également au jour un nouveau genos, les Euénorides].

54 MA John, « Dating the New Decree of the Confederation of Athena Ilias », EA 40 (2007), p. 55-57.

Emily, "A Boiotian Proxeny Decree and Relief in the Museum of Fine Arts, Boston and Boiotian-Lakonian Relations in the 360s ", Chiron 38 (2008), p. 157-194 [la stèle est sculptée de représentations d'Héraclès enfant terrassant les serpents (référence à Thèbes), des Dioscures (référence à Sparte), d'Athéna Aléa (référence à Tégée), autant d'éléments qui influencent la manière de lire le texte].

56 MAGNANI Massimo, « Le Baccanti di Teocrito e Cos », ZPE 164 (2008), p. 33-44 [hypothèse d'une localisation de l'exécution du poème à Cos, notamment fondée sur des arguments religieux].

57 MARCHETTI Patrick, « Les dieux et héros du dromos dorien I. Réflexions sur les références légendaires de l'espace civique de Sparte et d'Argos chez Pausanias », ARG 10 (2008), p. 85-113. 


$$
\begin{aligned}
& \text { Topograph } \\
& \text { p. } 1-31 .
\end{aligned}
$$

70 p. 51-83.

MARI Manuela, «Festa mobile. Nemea e i suoi giochi nella tradizione letteraria e nell'evidenza materiale. I: l'età arcaica e classica ", IncidAntico 6 (2008), p. 91-132 [reconstruction de l'histoire du sanctuaire de Némée en Argolide, entre le premier quart du vi ${ }^{\mathrm{e}}$ siècle av. J.-C., au moment de la fondation des jeux néméens, et la fin du IV siècle av. J.-C.; l'analyse des témoignages amène des réflexions plus générales sur les témoignages disponibles concernant les principaux sanctuaires de la Grèce antique].

MARINATOS Nanno, "The So-called Hell and Sinners in the Odyssey and Homeric Cosmology ", Numen 56 (2009), p. 185-197 [Ulysse ne descend pas dans l'Hadès, même s'il assiste à la punition des grands coupables; la cosmologie à l'œuvre dans la représentation de l'au-delà serait d'origine égyptienne et le cosmos divisé en mondes diurne et nocturne].

MASSA Francesco, «Dioniso e Apollo dal teatro attico alla cultura imperiale: i tratti salienti di un complesso quadro documentario ", Mythos 1 (2006-2007), p. 77-92 [examen de sources littéraires qui traitent de la relation entre Apollon et Dionysos; celle-ci s'avère fondée sur la rencontre, le partage et parfois le renversement des rôles].

MEULDER Marcel, « Dédale serviteur des trois fonctions », Mythos 1 (2006-2007), p. 49-75 [sur l'origine indo-européenne du mythe de Dédale].

MICHel C., « Hermes in der Odyssee », WüJbb 32 (2008), p. 11-34.

MILETTI Lorenzo, «Herodotus in Theon's Progymnasmata. The Confutation of Mythical Accounts ", MH 65 (2008), p. 65-76 [analyse de la manière très personnelle dont Théon aborde la critique des mythes et leur rationalisation au départ de sa réflexion rhétorique sur l'œuvre d'Hérodote].

MIRón Dolores, "The Heraia at Olympia: Gender and Place », AJAH 3-4 (2004-2005) [2007], p. 7-38 [les compétitions d'olympie permettaient de reproduire l'ordre social dans toutes ses implications : l'ordre des "genres » était renforcé par la réaffirmation $\mathrm{du}$ rôle de chaque sexe et quatre thèmes majeurs étaient célébrés, la guerre et la paix, la virginité irrationnelle et le mariage].

MORENo Alfonso, "Hieron. The Ancient Sanctuary at the Mouth of the Black Sea ", Hesperia 77 (2008), p. 655-709 [mise au point très complète sur le site qui mériterait des fouilles en bonne et due forme; dossier des sources littéraires et épigraphiques en annexe].

MOYER Ian S., « Notes on Re-Reading the Delian Aretalogy of Sarapis (IG XI.4 1299) », ZPE 166 (2008), p. 101-107.

MYLONOPOULOS Joannis, « Natur als Heiligtum - Natur im Heiligtum », ARG 10 (2008),

8 NADAL Eléonore, «Poséidon sur le passage d'Héraklès sur quelques vases attiques à figures noires ", Pallas 76 (2008), p. 31-50 [le rôle de Poséidon dans ces représentations est comparable à celui qu'il joue dans d'autres épisodes mythologiques où il permet un passage, un changement de statut].

9 NOwICKI Krzysztof, "Some Remarks on the New Peak Sanctuaries in Crete: The Topography of Ritual Areas and their Relationship with Settlements ", JDAI 122 (2007),

OLLER GUZMÁN Marta, "Quelques remarques à propos de deux nouvelles dédicaces à Thétis et Achille trouvées à Apollonia d'Illyrie », ZPE 167 (2008), p. 75-80 [le participe $\sigma u v \alpha \mu \varphi \iota \pi 0 \lambda \varepsilon v ́ \sigma \alpha \varsigma$ montre qu'on acceptait la participation au culte de plusieurs 
personnes, dont une au moins était une femme; en outre, le culte des Néréides pourrait trouver son origine à Corinthe et avoir suivi la voie de la colonisation archaïque].

PALAGIA Olga, «The Parthenon Frieze: Boy or Girl? », AK 51 (2007), p. 3-7 [arguments en faveur de l'identification du personnage associé à la remise du péplos d'Athéna comme étant une fille, alors que le garçon avait eu la faveur de bon nombre d'interprétations récentes]. PEPONI Anastasia-Erasmia, "Choreia and Aesthetics in the Homeric Hymn to Apollo: The Performance of the Delian Maidens (Lines 156-64)», Classical Antiquity 28 (2009), p. 39-70.

73 PIERRE Hélène, «Le voyage de Triptolème en Étrurie et en Grande Grèce », Pallas 77 (2008), p. 111-132 [l'étude de l'iconographie de Triptolème en Étrurie et en Grande Grèce démontre qu'il a conservé son aspect de héros civilisateur athénien hors des frontières de l'Attique et qu'il s'est de plus parfaitement inséré dans ces régions. Inventaire des documents]. PIERRE Hélène, «Réflexions autour de la Nesteia des Thesmophories athéniennes », Pallas 76 (2008), p. 85-94 [l'abstinence alimentaire et sexuelle ainsi que l'aischrologia des Thesmophories semblent avoir été un rituel d'inversion appelant un renouveau, un retour au civilisé]. PIRENNE-DELforge Vinciane, "Le lexique des lieux de culte dans la Périégèse de Pausanias », ARG 10 (2008), p. 143-178. PRÊTRE Clarisse, «Une mécène au service d'une déesse thasienne : décret pour Stilbôn (THANAR 1) », BCH 130 (2006), p. 487-497.

ReITZAMmER L., « Aristophanes' Adôniazousai », Classical Antiquity 27 (2008), p. 282-333 [un scholiaste prétend que la Lysistrata aurait pu s'appeler Adôniazousai, les femmes utilisant l'acropole comme " toit » pour " célébrer » la fête, ce qui conduit à nuancer la lecture de la pièce et à revoir la place des Adonies à Athènes, de même que la distinction « fêtes privées/fêtes publiques »]. RENAUD Jean-Michel, « Les mythes chez Homère et chez Pindare : la famille vue sous des éclairages différents ", Ateliers (Lille 3) 37 (2007), p. 25-34 (volume sur les Représentations mythologiques du sentiment familial : autour de la haine et de l'amour).

RHODES P.J., "State and religion in Athenian inscriptions ", G \& R 56 (2009), p. 1-13 [fondée sur l'épigraphie athénienne, cette mise au point souligne que la religion était une préoccupation des citoyens, qui la portaient donc dans les organes de la cité qui était la leur; ces organes n'étaient ni spécialement religieux, ni clairement séculiers; ils s'occupaient de religion de la même manière qu'ils traitaient les autres préoccupations des citoyens].

RosóŁ Rafał, « Die Herkunft des Gottesnamens Apollon », Glotta 83 (2007), p. 222-242 [en faveur d'une étymologie phénicienne].

RUNZA Roberta, "Tipologie rituali demetriache in Magna Grecia: la partecipazione maschile", Mythos 1 (2006-2007), p.93-116 [les cultes de Déméter constituent un système rituel spécifique dans lequel le rôle des hommes et des femmes est déterminé par leur contribution à l'affirmation des valeurs sociales et politiques célébrées par de tels cultes]. Kilikien », EA 40 (2007), p. 35-40. 

klassischen Athens ", JDAI 122 (2007), p. 51-79 [analyse des rares monuments funéraires qui représentent les défunts dans l'au-delà ou lors de leur passage d'un espace à l'autre].

SINEUX Pierre, «Dormir, rêver, montrer... À propos de quelques 'représentations figurées' du rite de l'incubation sur les reliefs votifs des sanctuaires guérisseurs de l'Attique ", Kentron 23 (2007), p. 11-29 [analyse de quelques célèbres reliefs liés à des guérisons, mis en rapport avec le contenu des iamata d'Épidaure qui datent de la même période].

TIBBE Conrad M., « Mädchen, Frauen, Göttinen. Lakonische weibliche Bronzestatuetten und Stützfiguren archaischer Zeit », MDAI(A) 122 (2007), p. 17-102 [catalogue et analyse de ces figures féminines, avec mise en évidence du pouvoir d'identification de celles qui ornaient les manches de miroir avec leurs utilisatrices].

STIBBE Conrad, "Laconian Bronzes from the Sanctuary of Apollo Hyperteleatas near Phoiniki (Laconia) and from the Acropolis of Athens ", BABesch 83 (2008), p. 17-45.

TORelli Mario, «Artemide Hemera a Poseidonia. Contributo alla ricostruzione del pantheon di una colonia achea", IncidAntico 6 (2008), p.11-47 [étude des correspondances possibles entre les cultes grecs originaux et les temples urbains encore existants de la colonie achéenne de Poseidonia].

TORJUSSEN Stian Sundell, "An Inscribed Gold Olive Leaf from Daphniotissa, near Elis ", ZPE 166 (2008), p. 151-152 [tablette qui semble avoir échappé aux récents corpus des «tablettes orphiques»; mise en perspective de l'homogénéité des documents péloponnésiens].

TZOUKALA Victoria, « Honorary Shares of Sacrificial Meat in Attic Vase Painting: Visual Signs of Distinction and Civic Identity », Hesperia 78 (2009), p. 1-40 [la représentation de la viande de pattes sur des vases de la fin $\mathrm{du} \mathrm{vI}^{\mathrm{e}}$ et $\mathrm{du} \mathrm{v}^{\mathrm{e}} \mathrm{s}$., en dehors de quelques scènes de boucherie, est interprétée comme l'illustration privilégiée de la notion de "part d'honneur " et peut dès lors être la marque d'une identité civique pour celui qui la reçoit].

WARNER SLANE Kathleen, "The End of the Sanctuary of Demeter and Kore on Acrocorinth », Hesperia 77 (2008), p. 465-496.

91 WILLI Andreas, «Frösche, Sünder, Initianden: Zu einem Aristophanischen Rätsel », MH 65 (2008), p. 193-211.

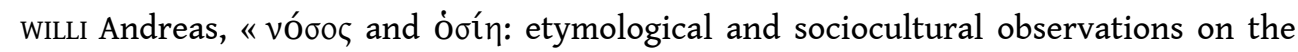
concepts of disease and divine (dis)favour in ancient Greece », JHS 128 (2008), p. 153-171 [sur la base de la reconstitution d'un non abstrait $*\left(h_{1}\right)$ osu, signifiant « bien-être », l'A. interprète óoín comme l'état de ce qui est caractérisé par ce bien-être, voulu par les dieux en raison de leur bienveillance; l'adjectif ǒøıৎৎ serait dès lors une formation secondaire fondée sur le nom, lié à la faveur divine et pouvant faire référence à ce qui est « profane »]. 